\title{
ZUR ETYMOLOGIE VON AE. MASSERE.
}

In seinem beitrag "Etymologisches und Grammatisches" in Anglia XXXI (XIX), 2. heft, s. $257 \mathrm{ff}$. gibt herr prof. A. Pogatscher eine erklärung der etymologie des ae. massere, kaufmann. Er leitet es ab von einem volkstümlichen, in der schriftsprache nicht zu findenden verb * massian, auf die messe gehen, das analog dem verhältnis bab : bapian sich aus ae. maesse, die messe (an die sich nachher handeln knüpft), entwickelt haben soll (neben der belegten form massian, die messe lesen). - Die anlehnung an messe lälst sich in der tat begrifflich leicht begründen mit hinweis auf die bedeutungsentwicklung des mlat. missa im mhd. mësse, gottesdienst feiertag - jahrmarkt.

Die erklärung ist aber dennoch nicht befriedigend. Wenn massere mit masse im zusammenhang stünde, so mülsten wir die bedeutung moesse, jahrmarkt oder händlerzusammenkunft, als das ältere wort, aus dem das fragliche *massian, zur messe gehen (d.h. zur handelsgelegenheit gehen) erst abzuleiten war, irgendwo im Ae. oder frühern Me. finden. Wiederholt ist in den angelsächsischen gesetzen vou handel auf märkten die rede. Immer aber wird das wort céap oder céapstów gebraucht und nie moesse. Das ae. moesse scheint nur die bedeutung 'kirchlicher gottesdienst' (mit mehreren unterbedeutungen, s. NED. unter mass) und kirchlicher festtag gehabt zu haben (wie die ausdrücke Christmas, Lammas usw. bezeugen und die formel Sunnandazum and moessedazum in Blickl. Hom. 47, s. NED. unter Mass-day). Von der merkantilen seite, die der kirchliche gottesdienst in späterer zeit aufweist, haben 
wir in der altenglischen periode kein zeugnis. ${ }^{1)}$ Ein gesetz verbietet sogar ausdrücklich das handeln an sonntagen, ${ }^{2}$ ) so dals in der volksseele der begriff festtag nicht so leicht mit kauftag identisch werden konnte. Eine verbindung der beiden begriffe gottesdienst und markt scheint erst in mittelenglischer zeit durch kontinentalen einflufs zu stande gekommen zu sein. Während die auf dem mittelenglischen markte herrschende sitte der bekräftigung von handänderungen durch das "trinkgeld", beveragium ${ }^{3}$ ) bis auf die altenglische zeit zurückgeführt werden kann (ae. drynceléan), ${ }^{4}$ ) finden wir bei den Angelsachsen noch keine spur von dem zur bindung von kaufverträgen zahlbaren God's-penny, denarius dei. ${ }^{5}$ ) Dieser kam der kirche zu gute. Sie sollte durch das brennen von heiligen kerzen auf einem altare (oder durch das lesen einer messe?) dem vertrag kirchliche sanktion verleihen. ${ }^{6}$ ) Die sitte war wahrscheinlich vom kontinente her importiert worden. ${ }^{7}$ ) In ihr erblicken wir eines jener spätern bindeglieder zwischen kirche und markt. In diesem zusammenhange mag auch erwähnt werden, dals die deutsche begriffsentwicklung messe - feiertag - jahrmarkt auch eine relativ späte ist und erst für das frühe 14. jahrhundert belegt werden kann. ${ }^{8}$ )

1) Wenn in der spätangelsächsischen zeit (1020-c. 1060) ein gesetz den marktplatz und die kirchengemeinde in einer formel zusammenschmiedet (Liebermann, Gesetze I, 400, Hit Becwæð: in ceapstowe oppe on cyricware), so ist es wohl der gemeinsame begriff öffentlichkeit, der anlals zur verbindung gibt.

2) Sunnandæges cypinge we forbeodar, Liebermann, I, 381, 55, Northnmbrisches Priestergesetz aus York.

s) S. Ducange, 8. v. biberagium. Ferner Select Cases concerning the Iavo Merchant, A. D. 1270-1638, vol. I, ed. C. Gross, Selden Society, 1908. S. 47 and Glossary.

4) Liebermann, Gesetze, I, 385, 67. Im wörterbuch erklärt als 'geld zam trinken bei handänderungen'.

s) 1291 : super quas forcipes solvit (d. h. der käufer) eidem (dem verkäufer) argentum dei et beveragium. Select Cases etc., s. 47. - Ferner beispiel in NED. unter God's-penny: 1340 Ayenb. 91 pe loue of herte, pet is pe godes peny huermide me bayp alle pe guodes of pe wordle.

6) F. W. Maitland, Select Pleas in Manorial and other Seignorial Courts, vol. 1, Selden Society, 1888, s. 133.

') F. W. Maitland, a. a. 0., s. 133.

8) M. Heyne in Grimm, Deutsches Wörterbuch, unter messe. 
Lautlich läIst sich bemerken, dafs wir ae. nicht maesse sondern messe (wie im Kentischen und Mercischen nach NED., und im Northumbrischen nach Kluge, Etym. Wrtb. der d. Spr.) ') erwarten sollten, dafs es deshalb gefährlich ist, wenn wir die abweichung von $e$ nach $a$ durch einen weitern schritt nach $a$ hin noch vergröfsern. Ferner ist zu beachten, dafs die kombination $a+s s$ nicht angelsächsisch ist, also nicht zu analogiebildungen verlocken konnte. Die wörter mit $a+s s$ im Ae., wenn - abgesehen von massere - solche ïberhaupt zu finden sind, dürften an den fingern abzuzählen sein. Die kombination $a+s s$ weist deshalb auf direkte entlehnung hin. Wie wir ae. mynetere auf lat. monetarius, tolnere auf tolonearius, sester auf sextarius zurückführen, so können wir auch aus ae. massere lat. *massarius rekonstruieren. Sehen wir bei Ducange nach, so finden wir ein wort massarius mit vielen bedeutungen, z. b. schatzmeister (fisci communis custos), und eine hinweisung auf das von der gleichen wortgruppe abgeleitete französische massart (s. auch Godefroi). Massarius, das in der bedeutung schatzmeister für das jahr 1366 belegt ist, hat auch den sinn von verwalter und 'Économe' (1311). Seine älteste bedeutung soll nach Ducange diejenige von pächter (colonus) und gutsvogt (villicus, massae custos, dem spätern englischen senescallus entsprechend) sein. Das wort ist abzuleiten aus massa $<$ masa < mansa, wobei mansa bezw. massa ähnlich wie ae. híd (land für eine familie, dann eine ideelle fiskalische mafseinheit) ${ }^{2}$ ) grundstïck und haus bedeutete, sowie auch eine bestimmte fläche land. ${ }^{3}$ ) Diesen sinn (grundstück und haus) hat das wort massa in den ältesten langobardischen urkunden (Ducange). Das $s s$ neben $s$ erklärt sich durch die qualitätsgleichheit von vulg. lat. $\bar{a}$ und $\breve{a}$. Aus massarius (von massa < mansa) mufste sich schon frïh die

1) Auffallend ist auch das überwiegen des $e$ gegen $a$ in den me. texten bis ungefähr 1500 , wo das $a$ allgemein wird. Chaucer hat $a$, aher Wyclif, Robert Brunne, Cursor M., Ancr. R. und Orm. (unter mass-book, NED.) haben alle $e$ (s. formen im NED.). (Vielleicht französischer einflufs.)

2) F. IV. Maitland, Domesday Book and Beyond, s. 391.

s) Vgl. dazu die in Maitland, Domesday etc. s. 359 erwähnten, an stelle des spätern hida (bevor das ae. híd latinisiert wurde) in englisch-lateinischen dokumenten gebrauchten ausdriicke: terra unius manentis, terra unius casati, später auch mansa, einige male mansio. 
bedeutung landwirt entwickeln, dann gutsvogt, rechnungsführer, ferner 'derjenige, der kaufen und verkaufen auf einem herrengute besorgt', 1) sehliefslich händler, kaufmann. Demnach wäre ae. massere durch den verkehr mit romanischen völkern ins Ae. eingeführt worden. ${ }^{2}$ )

Vielleicht liefse sich auch ae. massere mit lat. massa (masse) in verbindung bringen. Ducange belegt für 1159 masseria, welches waren aller art bedeuten soll (ob dieses masseria nicht auch von masa < mansa kommt als hausgerät usw.?). Godefroi erwähnt das entsprechende wort für das franz.: masserie als bagage, dem der sinn ware naheliegt. Wir mürsten dann ein mlat. massa = ware annehmen, aus dem sich *massarius gebildet hätte.

Korrekturnote. Seit der abfassung des obigen beitrages (Januar 1909) ist mir aufgefallen, dås in den Gesetzen (Lieberm. 458, H, 6) mássere notiert ist. Wenn das $a$ lang war, läfst sich in der tat ae. mässere mit māssarius (= māsarius, mānsarius) leicht zusammenstellen. $\mathrm{Zu}$ gleicher zeit verschwindet auch die möglichkeit einer analogie mit mcessere, massian (měssa, m̌ssa).

1) Ein beispiel dafür, wie ein ursprünglicher titel (wie massarius) zur bezeichnung einer berufart wird oder eine weitere bedeutung annimmt, wird durch ne. purveyor und durch das verb 'to cater' geliefert. Heute heifst purveyor li ef erant im allgemeinen, eine art kaufmann. Ursprïnglich ist der 'purveour' ein beamter des königlichen haushaltes, der die lebensmittel für den königlichen hof einkaufen mufs. Vgl. 1300, Statutes of the Realm, I, s. 137: purveours pur soen (d. h. des königs) Hostel. Ferner 1362, ibid. 371: les Purveours des vitailles del Hostel le Roi. - Im jahre 1362 (Statutes, s. 371) wird dieser titel umgeändert zu achatour resp. akatour. Dies gab ne. cater, proviantmeister (jetzt caterer), aus dem sich das $v b$. 'to cater' lebensmittel einkaufen bildete, das an den ursprünglichen titel nicht mehr erinnert.

2) Für die weite verbreitung der wortgruppe masa $<$ mansa spricht auch die tatsache, dals in noch ahd. zeit durch diese gruppe auch der deutschen sprache ein wort zugeführt worden ist: mansionarius $>$ masinarius $>$ mẹsinari $>$ mesnære $>$ Messner (Kluge, Et. Wtb.).

St. Gallen.

Bernhard Fehr. 